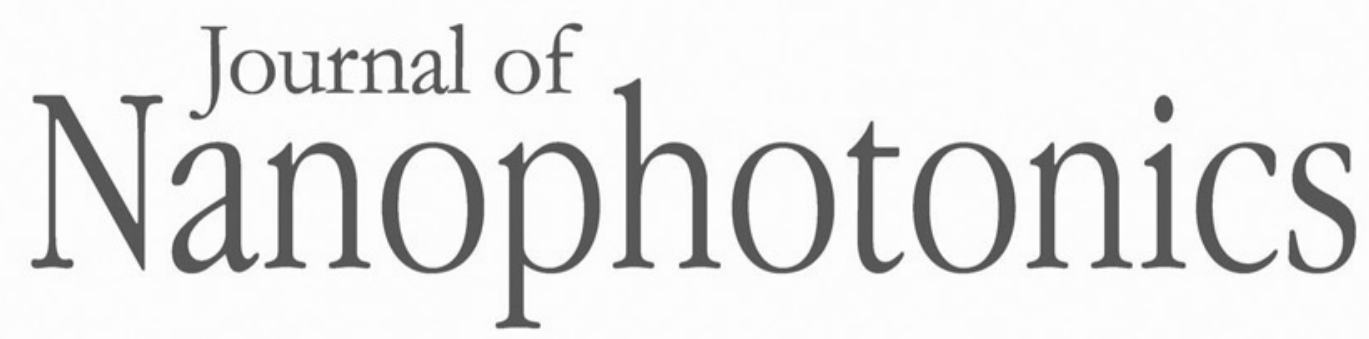

Nanophotonics.SPIEDigitalLibrary.org

\title{
Left/right asymmetry in reflection and transmission by a planar anisotropic dielectric slab with topologically insulating surface states
}

\author{
Akhlesh Lakhtakia \\ Tom G. Mackay
}




\title{
Left/right asymmetry in reflection and transmission by a planar anisotropic dielectric slab with topologically insulating surface states
}

\author{
Akhlesh Lakhtakia ${ }^{a}$; and Tom G. Mackay ${ }^{\mathrm{a}, \mathrm{b}}$ \\ aPennsylvania State University, Department of Engineering Science and Mechanics, \\ University Park, Pennsylvania 16802, United States \\ ${ }^{b}$ University of Edinburgh, School of Mathematics and Maxwell Institute for Mathematical \\ Sciences, Edinburgh EH9 3FD, Scotland, United Kingdom
}

\begin{abstract}
The reflection and transmission of plane waves by a homogeneous anisotropic dielectric slab-as represented by a columnar thin film - with topologically insulating surface states was theoretically investigated. Copolarized and cross-polarized reflectances and transmittances were calculated by solving the associated boundary-value problem. Numerical calculations revealed that all four reflectances and all four transmittances were asymmetric with respect to reversal of projection of the propagation direction of the incident plane wave on the illuminated surface of the slab. This left/right reflection and transmission asymmetry arises due to the combined effects of the slab's anisotropy and surface states. () The Authors. Published by SPIE under a Creative Commons Attribution 3.0 Unported License. Distribution or reproduction of this work in whole or in part requires full attribution of the original publication, including its DOI. [DOI: 10.1117/1.JNP.10.020501]
\end{abstract}

Keywords: admittance; columnar thin film; surface state; topological insulator.

Paper 16058L received Apr. 11, 2016; accepted for publication May 31, 2016; published online Jun. 22, 2016.

\section{Introduction}

The modest aim of this communication is to theoretically show that a homogeneous anisotropic dielectric slab with topologically insulating surface states (TISS) ${ }^{1,2}$ reflects and transmits light in such a way as to exhibit asymmetry with respect to reversal of projection of the propagation direction of the incident plane wave on the illuminated surface of the slab. We refer to this phenomenon as left/right asymmetry.

A homogeneous anisotropic material is characterized by a frequency-dependent relative permittivity dyadic $\underline{\underline{\varepsilon}}$. Suppose that this material occupies the region $\mathcal{V}_{\text {in }}$ bounded by the surface $\mathcal{S}$, which separates $\mathcal{V}_{\text {in }}$ from the vacuous region $\mathcal{V}_{\text {out }}$. If the anisotropic material possesses TISS, then the boundary conditions ${ }^{3}$

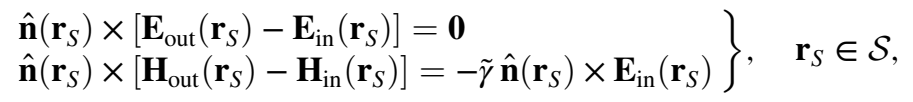

hold, with the unit normal vector $\hat{\mathbf{n}}\left(\mathbf{r}_{S}\right)$ at $\mathbf{r}_{S} \in \mathcal{S}$ pointing into $\mathcal{V}_{\text {out }}$ and the admittance $\tilde{\gamma}$ describing the TISS. Although optical scattering by isotropic dielectric materials, i.e., $\underline{\varepsilon}=$ $\varepsilon\left(\hat{\mathbf{u}}_{x} \hat{\mathbf{u}}_{x}+\hat{\mathbf{u}}_{y} \hat{\mathbf{u}}_{y}+\hat{\mathbf{u}}_{z} \hat{\mathbf{u}}_{z}\right)$, with TISS has been investigated theoretically ${ }^{4-7}$ as well as experimentally, ${ }^{2,8}$ this communication is possibly the first report of optical scattering by an anisotropic dielectric material with TISS. The existence of such materials is deemed possible because the isotropic materials with TISS are chalcogenides, ${ }^{1,2}$ columnar thin films (CTFs) of other chalcogenides have been fabricated, ${ }^{9}$ and CTFs function as anisotropic dielectric materials at sufficiently low frequencies. ${ }^{10}$ Furthermore, periodically multilayered composite materials ${ }^{11,12}$ comprising laminas of an isotropic topological insulator and some other material should function as effectively anisotropic continuums at sufficiently low frequencies. ${ }^{13}$

*Address all correspondence to: Akhlesh Lakhtakia, E-mail: akhlesh@psu.edu 
We have found that the TISS induce the exhibition of left/right asymmetry in reflection and transmission by a homogeneous anisotropic dielectric slab. This asymmetry could be exploited for one-way optical devices. The boundary-value problem of reflection and transmission of an obliquely incident plane wave by a homogeneous anisotropic dielectric slab with TISS is described and solved in Sec. 2. Illustrative numerical results are presented and discussed in Sec. 3.

The free-space wavenumber, the free-space wavelength, and the intrinsic impedance of free space are denoted by $k_{0}=\omega \sqrt{\varepsilon_{0} \mu_{0}}, \lambda_{0}=2 \pi / k_{0}$, and $\eta_{0}=\sqrt{\mu_{0} / \varepsilon_{0}}$, respectively, with $\mu_{0}$ and $\varepsilon_{0}$ being the permeability and permittivity of free space. We denote the fine structure constant by $\tilde{\alpha}=\left(q_{e}^{2} \eta_{0}\right) / 2 \tilde{h}$, where $q_{e}$ is the quantum of charge and $\tilde{h}$ is the Planck constant. Vectors are in boldface, dyadics are underlined twice, column vectors are in boldface and enclosed within square brackets, while matrixes are underlined twice and similarly bracketed. Cartesian unit vectors are identified as $\hat{\mathbf{u}}_{x}, \hat{\mathbf{u}}_{y}$, and $\hat{\mathbf{u}}_{z}$.

\section{Theory}

Suppose that the regions $\mathcal{V}_{\text {in }}=\{(x, y, z): z \in(0, L)\}$ and $\mathcal{V}_{\text {out }}=\{(x, y, z): z \notin[0, L]\}$ are separated by the surface $\mathcal{S}=\{(x, y, z): z \in\{0, L\}\}$.

A plane wave, propagating in the half-space $z<0$ at an angle $\theta \in[0, \pi / 2)$ to the $z$-axis and at an angle $\psi \in[0,2 \pi)$ to the $x$-axis in the $x y$ plane, is incident on the slab, as shown in Fig. 1 . The electromagnetic field phasors associated with the incident plane wave are represented as

$$
\left.\begin{array}{l}
\mathbf{E}_{\text {inc }}(r)=\left(a_{s} \mathbf{s}+a_{p} \mathbf{p}_{+}\right) \exp \left[i \kappa(x \cos \psi+y \sin \psi)+i k_{0} z \cos \theta\right] \\
\mathbf{H}_{\text {inc }}(r)=\frac{1}{\eta_{0}}\left(a_{s} \mathbf{p}_{+}-a_{p} \mathbf{s}\right) \exp \left[i \kappa(x \cos \psi+y \sin \psi)+i k_{0} z \cos \theta\right]
\end{array}\right\}, \quad z<0 .
$$

The amplitudes of the $s$ - and the $p$-polarized components of the incident plane wave, denoted by $a_{s}$ and $a_{p}$, respectively, are assumed given, whereas

$$
\left.\begin{array}{l}
\kappa=k_{0} \sin \theta, \quad \mathbf{s}=-\hat{\mathbf{u}}_{x} \sin \psi+\hat{\mathbf{u}}_{y} \cos \psi \\
\mathbf{p}_{ \pm}=\mp\left(\hat{\mathbf{u}}_{x} \cos \psi+\hat{\mathbf{u}}_{y} \sin \psi\right) \cos \theta+\hat{\mathbf{u}}_{z} \sin \theta
\end{array}\right\}
$$

The reflected electromagnetic field phasors are expressed as

$$
\left.\begin{array}{l}
\mathbf{E}_{\mathrm{ref}}(r)=\left(r_{s} \mathbf{s}+r_{p} \mathbf{p}_{-}\right) \exp \left[i \kappa(x \cos \psi+y \sin \psi)-i k_{0} z \cos \theta\right] \\
\mathbf{H}_{\mathrm{ref}}(r)=\frac{1}{\eta_{0}}\left(r_{s} \mathbf{p}_{-}-r_{p} \mathbf{s}\right) \exp \left[i \kappa(x \cos \psi+y \sin \psi)-i k_{0} z \cos \theta\right]
\end{array}\right\}, \quad z<0,
$$

and the transmitted electromagnetic field phasors as

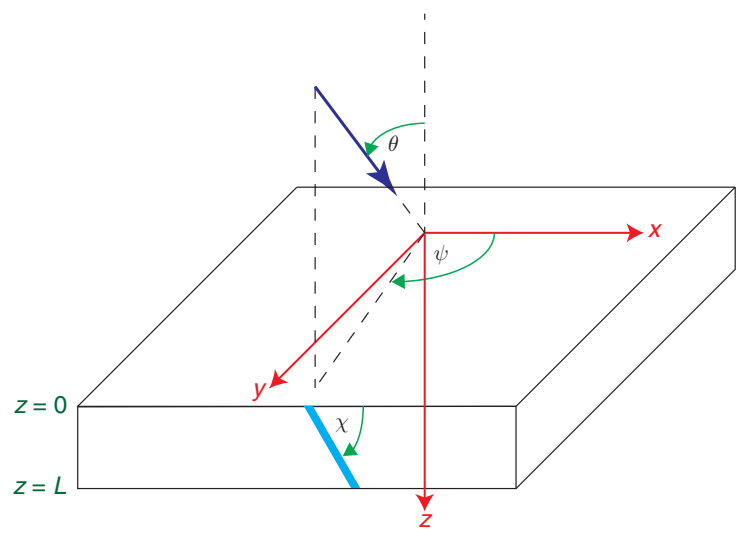

Fig. 1 A plane wave is incident on the slab $\mathscr{V}_{\text {in }}=\{(x, y, z): z \in(0, L)\}$; the wave vector of the incident plane wave inclined at an angle $\theta$ with respect to the $z$-axis and at an angle $\psi$ with respect to the $x$-axis in the $x y$ plane. Also shown is the angle $\chi$. 


$$
\left.\begin{array}{l}
\mathbf{E}_{\mathrm{tr}}(r)=\left(t_{s} \mathbf{s}+t_{p} \mathbf{p}_{+}\right) \exp \left[i \kappa(x \cos \psi+y \sin \psi)+i k_{0}(z-L) \cos \theta\right] \\
\mathbf{H}_{\mathrm{tr}}(r)=\frac{1}{\eta_{0}}\left(t_{s} \mathbf{p}_{+}-t_{p} \mathbf{s}\right) \exp \left[i \kappa(x \cos \psi+y \sin \psi)+i k_{0}(z-L) \cos \theta\right]
\end{array}\right\}, \quad z>L .
$$

The reflection amplitudes $r_{s}$ and $r_{p}$, as well as the transmission amplitudes $t_{s}$ and $t_{p}$, have to be determined by the solution of a boundary-value problem.

The frequency-domain electromagnetic constitutive relations of the homogeneous anisotropic dielectric material in $\mathcal{V}_{\text {in }}$ can be written as ${ }^{10}$

$$
\mathbf{D}(\mathbf{r})=\varepsilon_{0} \underline{\underline{\varepsilon}} \cdot \mathbf{E}(\mathbf{r}), \quad \mathbf{B}(\mathbf{r})=\mu_{0} \mathbf{H}(\mathbf{r}), \quad z \in(0, L),
$$

where the dyadics

$$
\begin{gathered}
\underline{\underline{\varepsilon}}=\underline{\underline{S}}_{y} \cdot\left(\varepsilon_{a} \hat{\mathbf{u}}_{z} \hat{\mathbf{u}}_{z}+\varepsilon_{b} \hat{\mathbf{u}}_{x} \hat{\mathbf{u}}_{x}+\varepsilon_{c} \hat{\mathbf{u}}_{y} \hat{\mathbf{u}}_{y}\right) \cdot \underline{\underline{S}}_{y}^{T}, \\
\underline{\underline{S}}_{y}=\left(\hat{\mathbf{u}}_{x} \hat{\mathbf{u}}_{x}+\hat{\mathbf{u}}_{z} \hat{\mathbf{u}}_{z}\right) \cos \chi+\left(\hat{\mathbf{u}}_{z} \hat{\mathbf{u}}_{x}-\hat{\mathbf{u}}_{x} \hat{\mathbf{u}}_{z}\right) \sin \chi+\hat{\mathbf{u}}_{y} \hat{\mathbf{u}}_{y}
\end{gathered}
$$

involve the angle $\chi \in[0, \pi / 2]$. The superscript ${ }^{T}$ denotes the transpose. The principal relative permittivity scalars $\varepsilon_{a}, \varepsilon_{b}$, and $\varepsilon_{c}$, as well as the angle $\chi$, can be chosen for application to either natural crystals ${ }^{14}$ or the manufactured CTFs. ${ }^{10}$

In $\mathcal{V}_{\text {in }}$, the electric and magnetic field phasors can be represented as ${ }^{15}$

$$
\left.\begin{array}{l}
\mathbf{E}(\mathbf{r})=\mathbf{e}(z) \exp [i \kappa(x \cos \psi+y \sin \psi)] \\
\mathbf{H}(\mathbf{r})=\mathbf{h}(z) \exp [i \kappa(x \cos \psi+y \sin \psi)]
\end{array}\right\},
$$

where the vector functions $\mathbf{e}(z)$ and $\mathbf{h}(z)$ are unknown. Substitution of Eqs. (6) and (9) in the Maxwell curl postulates followed by certain algebraic manipulations leads to the $4 \times 4$-matrix ordinary differential equations

$$
\frac{d}{d z}[\mathbf{f}(z)]=i[\underline{\underline{P}}] \cdot[\mathbf{f}(z)], \quad z \in \mathcal{V}_{\mathrm{in}},
$$

where the column vector

$$
[\mathbf{f}(z)]=\left[e_{x}(z), e_{y}(z), h_{x}(z), h_{y}(z)\right]^{T},
$$

the $4 \times 4$ matrix

$$
\begin{aligned}
{[\underline{\underline{P}}]=} & \omega\left[\begin{array}{cccc}
0 & 0 & 0 & \mu_{0} \\
0 & 0 & -\mu_{0} & 0 \\
0 & -\varepsilon_{0} \varepsilon_{c} & 0 & 0 \\
\varepsilon_{0} \varepsilon_{d} & 0 & 0 & 0
\end{array}\right]+\kappa \frac{\varepsilon_{d}\left(\varepsilon_{a}-\varepsilon_{b}\right)}{2 \varepsilon_{a} \varepsilon_{b}} \sin (2 \chi)\left[\begin{array}{cccc}
\cos \psi & 0 & 0 & 0 \\
\sin \psi & 0 & 0 & 0 \\
0 & 0 & 0 & 0 \\
0 & 0 & -\sin \psi & \cos \psi
\end{array}\right] \\
& +\frac{\kappa^{2}}{\omega \varepsilon_{0}} \frac{\varepsilon_{d}}{\varepsilon_{a} \varepsilon_{b}}\left[\begin{array}{cccc}
0 & 0 & \cos \psi \sin \psi & -\cos ^{2} \psi \\
0 & 0 & \sin ^{2} \psi & -\cos \psi \sin \psi \\
0 & 0 & 0 & 0 \\
0 & 0 & 0 & 0
\end{array}\right] \\
& +\frac{\kappa^{2}}{\omega \mu_{0}}\left[\begin{array}{cccc}
0 & 0 & 0 & 0 \\
0 & 0 & 0 & 0 \\
-\cos \psi \sin \psi & \cos ^{2} \psi & 0 & 0 \\
-\sin ^{2} \psi & \cos \psi \sin \psi & 0 & 0
\end{array}\right]
\end{aligned}
$$

and the scalar

$$
\varepsilon_{d}=\frac{\varepsilon_{a} \varepsilon_{b}}{\varepsilon_{a} \cos ^{2} \chi+\varepsilon_{b} \sin ^{2} \chi} .
$$

Equation (10) has the following straightforward solution:

$$
\left[\mathbf{f}\left(L^{-}\right)\right]=\exp \{i[\underline{\underline{P}}] L\} \cdot\left[\mathbf{f}\left(0^{+}\right)\right],
$$


where the notation $\left[\mathbf{f}\left(a^{ \pm}\right)\right]$stands for $\lim _{\delta \rightarrow 0}[\mathbf{f}(a \pm \delta)]$ with $\delta \nless 0$. Application of the boundary conditions [Eq. (1)] to the planes $z=0$ and $z=L$ leads to

$$
\begin{aligned}
& {\left[\mathbf{f}\left(0^{-}\right)\right]=\left[\underline{\underline{V}} \cdot\left[\mathbf{f}\left(0^{+}\right)\right],\right.} \\
& {\left[\mathbf{f}\left(L^{+}\right)\right]=\left[\underline{\underline{V}} \cdot\left[\mathbf{f}\left(L^{-}\right)\right],\right.}
\end{aligned}
$$

respectively, where the matrix

$$
[\underline{\underline{V}}]=\left[\begin{array}{cccc}
1 & 0 & 0 & 0 \\
0 & 1 & 0 & 0 \\
-\tilde{\gamma} & 0 & 1 & 0 \\
0 & -\tilde{\gamma} & 0 & 1
\end{array}\right]
$$

Combining Eqs. (14)-(16), we get

$$
\left[\mathbf{f}\left(L^{+}\right)\right]=[\underline{\underline{V}}] \cdot \exp \left\{i \left[\underline{\underline{P}}[L\} \cdot[\underline{\underline{V}}]^{-1} \cdot\left[\mathbf{f}\left(0^{-}\right)\right] .\right.\right.
$$

But the elements of $\left[\mathbf{f}\left(0^{-}\right)\right]$are known by virtue of Eqs. (2) and (4), and those of $\left[\mathbf{f}\left(L^{+}\right)\right]$by virtue of Eq. (5). Accordingly, Eq. (18) may be written as

$$
\left[\begin{array}{c}
t_{s} \\
t_{p} \\
0 \\
0
\end{array}\right]=[\underline{\underline{K}}]^{-1} \cdot[\underline{\underline{V}}] \cdot \exp \{i[\underline{\underline{P}}] L\} \cdot[\underline{\underline{V}}]^{-1} \cdot[\underline{\underline{K}}] \cdot\left[\begin{array}{c}
a_{s} \\
a_{p} \\
r_{s} \\
r_{p}
\end{array}\right],
$$

where

$$
[\underline{\underline{K}}]=\left[\begin{array}{cccc}
-\sin \psi & -\cos \psi \cos \theta & -\sin \psi & \cos \psi \cos \theta \\
\cos \psi & -\sin \psi \cos \theta & \cos \psi & \sin \psi \cos \theta \\
-\left(\frac{1}{\eta_{0}}\right) \cos \psi \cos \theta & \left(\frac{1}{\eta_{0}}\right) \sin \psi & \left(\frac{1}{\eta_{0}}\right) \cos \psi \cos \theta & \left(\frac{1}{\eta_{0}}\right) \sin \psi \\
-\left(\frac{1}{\eta_{0}}\right) \sin \psi \cos \theta & -\left(\frac{1}{\eta_{0}}\right) \cos \psi & \left(\frac{1}{\eta_{0}}\right) \sin \psi \cos \theta & -\left(\frac{1}{\eta_{0}}\right) \cos \psi
\end{array}\right] .
$$

The solution of Eq. (19) yields the reflection and transmission coefficients that appear as the elements of the $2 \times 2$ matrixes in the following relations:

$$
\left[\begin{array}{l}
r_{s} \\
r_{p}
\end{array}\right]=\left[\begin{array}{ll}
r_{s s} & r_{s p} \\
r_{p s} & r_{p p}
\end{array}\right]\left[\begin{array}{l}
a_{s} \\
a_{p}
\end{array}\right], \quad\left[\begin{array}{l}
t_{s} \\
t_{p}
\end{array}\right]=\left[\begin{array}{cc}
t_{s s} & t_{s p} \\
t_{p s} & t_{p p}
\end{array}\right]\left[\begin{array}{l}
a_{s} \\
a_{p}
\end{array}\right]
$$

Copolarized coefficients have both subscripts identical, but cross-polarized coefficients do not. The square of the magnitude of a reflection or transmission coefficient is the corresponding reflectance or transmittance; thus, $R_{s p}=\left|r_{s p}\right|^{2}$ is the reflectance corresponding to the reflection coefficient $r_{s p}$, and so on. The principle of conservation of energy mandates the constraints $R_{s s}+R_{p s}+T_{s s}+T_{p s} \leq 1$ and $R_{p p}+R_{s p}+T_{p p}+T_{s p} \leq 1$. Let us note here that a real-valued $\tilde{\gamma}$ does not cause dissipation.

\section{Numerical Results and Discussion}

Let the left side of the $x y$ plane be specified by $\psi \in[0, \pi]$ and the right side by $\psi \in[\pi, 2 \pi]$. In order to delineate the characteristics of and the factors responsible for left/right asymmetry of reflection and transmission, we need to consider four distinct cases as follows.

Case I: Suppose that $\varepsilon_{a}=\varepsilon_{b}=\varepsilon_{c}$ and $\tilde{\gamma}=0$. Then the material in $\mathcal{V}_{\text {in }}$ is a homogeneous isotropic dielectric material and the TISS are absent. The boundary-value problem then turns into a textbook reflection/transmission problem. ${ }^{16}$ None of the four reflectances $\left(R_{s s}, R_{p s}, R_{p p}\right.$, and $\left.R_{s s}\right)$ and the four transmittances $\left(T_{s s}, T_{p s}, T_{p p}\right.$, and $\left.T_{s s}\right)$ then depend on $\psi$. Furthermore, the cross-polarized remittances are null valued. In other words, the following relationships hold: 


$$
\begin{aligned}
& R_{s s}(\theta, \psi)=R_{s s}(\theta, 0), \quad R_{p p}(\theta, \psi)=R_{p p}(\theta, 0) \\
& \left.T_{s s}(\theta, \psi)=T_{s s}(\theta, 0), \quad T_{p p}(\theta, \psi)=T_{p p}(\theta, 0) \quad\right\} \text {. } \\
& \left.R_{p s}(\theta, \psi)=R_{s p}(\theta, \psi) \equiv 0, \quad T_{p s}(\theta, \psi)=T_{s p}(\theta, \psi) \equiv 0\right\}
\end{aligned}
$$

In particular, all reflectances and transmittances are unchanged upon replacing $\psi$ with $\psi+\pi$; i.e., all are left/right symmetric.

Case II: Suppose next that $\varepsilon_{a}=\varepsilon_{b}=\varepsilon_{c}$ but $\tilde{\gamma} \neq 0$. Then the material in $\mathcal{V}_{\text {in }}$ is a homogeneous isotropic dielectric material with TISS. None of the eight remittances $\left(R_{s s}\right.$, and so on, and $T_{s s}$, and so on) then depend on $\psi$, and the cross-polarized remittances are not identically zero. Analysis of numerical results reveals that the following relationships hold:

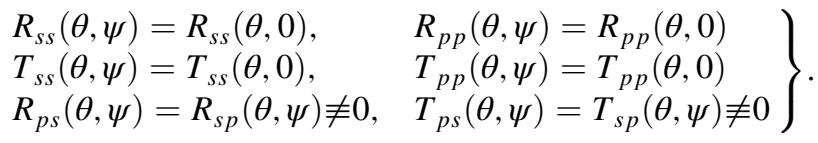

A comparison of Eqs. (22) and (23) indicates that the TISS are responsible for de-polarization on both reflection and transmission. As in case I, all eight remittances are left/right symmetric.

Case III: Suppose that $\varepsilon_{a}, \varepsilon_{b}$, and $\varepsilon_{c}$ are all dissimilar, but $\tilde{\gamma}=0$. Then the material in $\mathcal{V}_{\text {in }}$ is a homogeneous anisotropic dielectric material and the TISS are absent. Calculations then show the following symmetries:

$$
\left.\begin{array}{ll}
R_{s s}(\theta, \psi)=R_{s s}(\theta, \psi+\pi), & R_{p p}(\theta, \psi)=R_{p p}(\theta, \psi+\pi) \\
R_{p s}(\theta, \psi)=R_{s p}(\theta, \psi+\pi) \neq 0, & T_{p s}(\theta, \psi)=T_{s p}(\theta, \psi) \not \equiv 0
\end{array}\right\}
$$

While both $R_{s s}$ and $R_{p p}$ are left/right symmetric, both $T_{s s}$ and $T_{p p}$ are not. Furthermore, as $R_{p s}(\theta, \psi) \neq R_{s p}(\theta, \psi)$ and $T_{p s}(\theta, \psi) \neq T_{s p}(\theta, \psi+\pi)$, it follows that all cross-polarized remittances are left/right asymmetric. In summary, the following inequalities are entirely due to anisotropy:

$$
\left.\begin{array}{ll}
R_{p s}(\theta, \psi) \neq R_{p s}(\theta, \psi+\pi), & R_{s p}(\theta, \psi) \neq R_{s p}(\theta, \psi+\pi) \\
T_{s s}(\theta, \psi) \neq T_{s s}(\theta, \psi+\pi), & T_{p p}(\theta, \psi) \neq T_{p p}(\theta, \psi+\pi) \\
T_{p s}(\theta, \psi) \neq T_{p s}(\theta, \psi+\pi), & T_{s p}(\theta, \psi) \neq T_{s p}(\theta, \psi+\pi)
\end{array}\right\} .
$$

Case IV: Finally, $\varepsilon_{a}, \varepsilon_{b}$, and $\varepsilon_{c}$ are all dissimilar and $\tilde{\gamma} \neq 0$, so that the material in $\mathcal{V}_{\text {in }}$ is a homogeneous anisotropic dielectric material with TISS. All eight remittances then depend on $\psi$, the cross-polarized remittances are not identically zero, and only one relationship can be found.

$$
T_{p s}(\theta, \psi)=T_{s p}(\theta, \psi) \not \equiv 0 .
$$

All eight remittances exhibit left/right asymmetry, i.e.,

$$
\left.\begin{array}{ll}
R_{s s}(\theta, \psi) \neq R_{s s}(\theta, \psi+\pi), & R_{p p}(\theta, \psi) \neq R_{p p}(\theta, \psi+\pi) \\
R_{p s}(\theta, \psi) \neq R_{p s}(\theta, \psi+\pi), & R_{s p}(\theta, \psi) \neq R_{s p}(\theta, \psi+\pi) \\
T_{s s}(\theta, \psi) \neq T_{s s}(\theta, \psi+\pi), & T_{p p}(\theta, \psi) \neq T_{p p}(\theta, \psi+\pi) \\
T_{p s}(\theta, \psi) \neq T_{p s}(\theta, \psi+\pi), & T_{s p}(\theta, \psi) \neq T_{s p}(\theta, \psi+\pi)
\end{array}\right\} .
$$

Furthermore, by comparison with cases I to III, we deduce that these inequalities arise due to the combined effects of the slab's anisotropy and the presence of TISS. Without anisotropy, cases I and II show that the TISS are responsible for the cross-polarized reflectances and transmittances, but do not give rise to left/right asymmetry. Without TISS, case III shows that only the two copolarized transmittances (out of the eight remittances) are left/right asymmetric when the slab is made of an anisotropic material.

The complete left/right asymmetry that arises for case IV is illustrated in Figs. 2 and 3, wherein all reflectances and transmittances, respectively, are plotted as functions of the incidence angles $\theta \in[0, \pi / 2)$ and $\psi \in[0,2 \pi)$. For these representative calculations, we chose $\varepsilon_{a}=2.14$, 

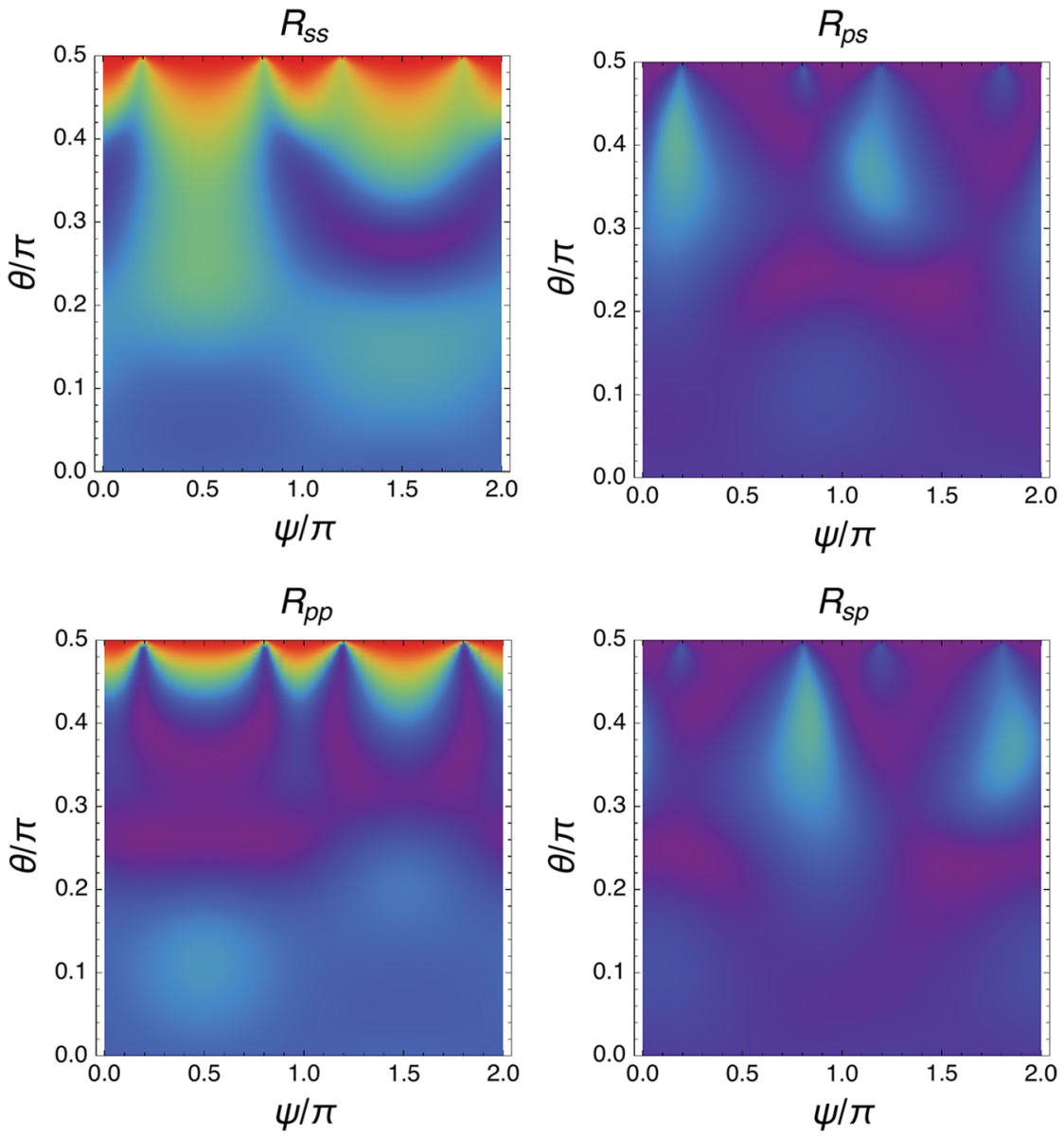

Fig. 2 Reflectances $R_{s s}, R_{p s}, R_{p p}$, and $R_{s p}$ as functions of the incidence angles $\theta \in[0, \pi / 2)$ and $\psi \in[0,2 \pi)$ when $\varepsilon_{a}=2.14, \varepsilon_{b}=3.67, \varepsilon_{c}=2.83, \chi=38 \mathrm{deg}, \tilde{\gamma}=100 \tilde{\alpha} / \eta_{0}$, and $L=1.4 \lambda_{0}$. The color coding employs the spectrum of the rainbow with the deepest violet denoting 0 and the deepest red denoting 1.0.

$\varepsilon_{b}=3.67, \varepsilon_{c}=2.83, \chi=38 \mathrm{deg}, \tilde{\gamma}=100 \tilde{\alpha} / \eta_{0}$, and $L=1.4 \lambda_{0}$. The chosen values of $\varepsilon_{a, b, c}$ emerged from a homogenization model for dielectric CTFs, ${ }^{15}$ whereas $\tilde{\gamma}^{17-19}$ was chosen to clearly highlight left/right asymmetry, in the absence of any experimental data for anisotropic topological insulators.

The inequalities in Eq. (27) are readily observed in the two figures. The left/right asymmetry is most easily discernible in the plots of $R_{s s}$ (Fig. 2) and $T_{p p}$ (Fig. 3), but can be identified in the plots of the remaining six remittances too for mid-range values of $\theta$.

In further numerical calculations (not presented here), the left/right asymmetry was found to be even more conspicuous for various remittances, when the magnitude of $\tilde{\gamma}$ was increased. Intrinsic topological insulators are characterized by $\tilde{\gamma}= \pm \tilde{\alpha} / \eta_{0},{ }^{1}$ but a very thin coating of a magnetic material can be used to realize $\tilde{\gamma}=(2 q+1) \tilde{\alpha} / \eta_{0}, q \in\{0, \pm 1, \pm 2, \pm 3, \ldots\} .{ }^{17,18}$ Values of $q$ other than -1 and 0 can also be obtained by immersing a topological insulator in a magnetostatic field. ${ }^{19}$

Practically oriented research on topological insulators is embryonic though steady progress is being made in the identification of several relevant materials. ${ }^{2,20}$ As stated in Sec. 1, attention is chiefly being given to isotropic topological insulators, although the fabrication of anisotropic topological insulators appears possible. The exploitation of left/right asymmetry theoretically shown here to be possible with anisotropic topological insulators is promising for one-way optical devices, which could reduce backscattering noise $\mathrm{e}^{21}$ in optical communication networks, microscopy, and tomography, for example. But high magnitudes of $\eta_{0} \tilde{\gamma} / \tilde{\alpha}$ would be needed for practical implementation. 

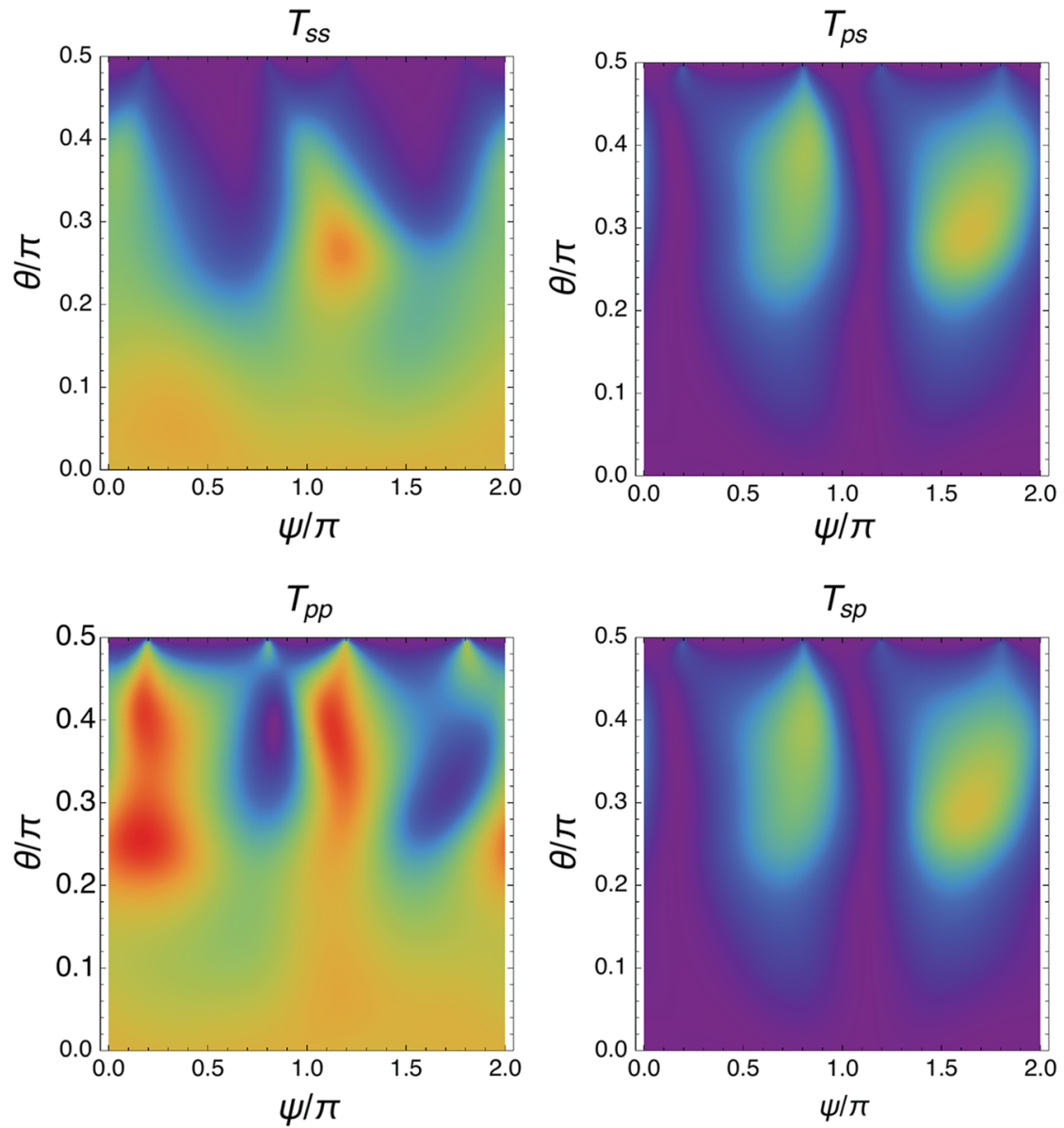

Fig. 3 Same as Fig. 2, except that the transmittances $T_{s s}, T_{p s}, T_{p p}$, and $T_{s p}$ are displayed as functions of $\theta$ and $\psi$.

\section{Acknowledgments}

A.L. is grateful to the Charles Godfrey Binder Endowment at Penn State for the ongoing support of his research. T.G.M. acknowledges the support of EPSRC Grant EP/M018075/1.

\section{References}

1. M. Z. Hasan and C. L. Kane, "Topological insulators," Rev. Modern Phys. 82(4), 30453067 (2010).

2. P. Di Pietro, Optical Properties of Bismuth-Based Topological Insulators, Springer, Cham, Switzerland (2014).

3. A. Lakhtakia and T. G. Mackay, "Classical electromagnetic model of surface states in topological insulators," J. Nanophoton. 10(3), 033004 (2016).

4. M.-C. Chang and M.-F. Yang, "Optical signature of topological insulators," Phys. Rev. B 80(11), 113304 (2009).

5. F. Liu et al., "Goos-Hänchen and Imbert-Fedorov shifts at the interface of ordinary dielectric and topological insulator," J. Opt. Soc. Am. B 30(5), 735-741 (2013).

6. F. Liu, J. Xu, and Y. Yang, "Polarization conversion of reflected electromagnetic wave from topological insulator," J. Opt. Soc. Am. B 31(4), 735-741 (2014).

7. A. Lakhtakia and T. G. Mackay, "Electromagnetic scattering by homogeneous, isotropic, dielectric-magnetic sphere with topologically insulating surface states," J. Opt. Soc. Am. B 33(4), 603-609 (2016). 
8. A. D. LaForge et al., "Optical characterization of $\mathrm{Bi}_{2} \mathrm{Se}_{3}$ in a magnetic field: infrared evidence for magnetoelectric coupling in a topological insulator material," Phys. Rev. B 81(12), 125120 (2010).

9. R. J. Martín-Palma et al., "Retardance of chalcogenide thin films grown by the obliqueangle-deposition technique," Thin Solid Films 517(10), 5553-5556 (2009).

10. I. J. Hodgkinson and Q. H. Wu, Birefringent Thin Films and Polarizing Elements, World Scientific, Singapore (1997).

11. F. Abelès, "Recherches sur la propagation des ondes électromagnétiques sinusoïdales dans les milieux stratifiés. Application aux couches minces (partie)," Ann. Phys. (Paris) 5, 596640 (1950).

12. P. W. Baumeister, Optical Coating Technology, SPIE, Bellingham, Washington (2004).

13. A. Lakhtakia, "Constraints on effective constitutive parameters of certain bianisotropic laminated composite materials," Electromagnetics 29(6), 508-514 (2009).

14. C. D. Gribble and A. J. Hall, Optical Mineralogy: Principles and Practice, University College Press, London, United Kingdom (1993).

15. A. Lakhtakia and R. Messier, Sculptured Thin Films: Nanoengineered Morphology and Optics, SPIE Press, Bellingham, Washington (2005).

16. M. Born and E. Wolf, Principles of Optics, 7th (expanded) ed., Cambridge University Press, Cambridge, United Kingdom (1999).

17. X.-L. Qi, T. L. Hughes, and S.-C. Zhang, "Topological field theory of time-reversal invariant insulators," Phys. Rev. B 78(19), 195424 (2008).

18. X.-L. Qi, T. L. Hughes, and S.-C. Zhang, "Erratum: topological field theory of time-reversal invariant insulators [Phys. Rev. B 78, 195424 (2008)]," Phys. Rev. B 81(15), 155901 (2010).

19. J. Maciejko et al., "Topological quantization in units of the fine structure constant," Phys. Rev. Lett. 105(16), 166803 (2010).

20. A. Amaricci et al., "First-order character and observable signatures of topological quantum phase transitions," Phys. Rev. Lett. 114(18), 185701 (2015).

21. C. Sayrin et al., "Nanophotonic optical isolator controlled by the internal state of cold atoms," Phys. Rev. X 5(4), 041036 (2015).

Akhlesh Lakhtakia received degrees from the Banaras Hindu University and the University of Utah. He is the Charles Godfrey Binder Professor of Engineering Science and Mechanics at the Pennsylvania State University. His research interests include surface multiplasmonics, biorepli-cation, forensic science, solar energy, sculptured thin films, and mimumes. He is a fellow of OSA, SPIE, IoP, AAAS, APS, and IEEE. He received the 2010 SPIE Technical Achievement Award and the 2016 Walston Chubb Award for Innovation.

Tom G. Mackay is a reader in the School of Mathematics at the University of Edinburgh and an adjunct professor in the Department of Engineering Science and Mechanics at the Pennsylvania State University. He is a graduate of the Universities of Edinburgh, Glasgow, and Strathclyde, and a fellow of the Institute of Physics (UK) and SPIE. His research interests include the electromagnetic theory of novel and complex materials, including homogenized composite materials. 